\title{
Tunable true-time delay of a microwave photonic signal realized by cross gain
} modulation in a semiconductor waveguide

\author{
Xue, Weiqi; Mørk, Jesper
}

Published in:

Applied Physics Letters

Link to article, DOI:

10.1063/1.3665946

Publication date:

2011

Document Version

Publisher's PDF, also known as Version of record

Link back to DTU Orbit

Citation (APA):

Xue, W., \& Mørk, J. (2011). Tunable true-time delay of a microwave photonic signal realized by cross gain modulation in a semiconductor waveguide. Applied Physics Letters, 99(23), 231102.

https://doi.org/10.1063/1.3665946

\section{General rights}

Copyright and moral rights for the publications made accessible in the public portal are retained by the authors and/or other copyright owners and it is a condition of accessing publications that users recognise and abide by the legal requirements associated with these rights.

- Users may download and print one copy of any publication from the public portal for the purpose of private study or research.

- You may not further distribute the material or use it for any profit-making activity or commercial gain

- You may freely distribute the URL identifying the publication in the public portal

If you believe that this document breaches copyright please contact us providing details, and we will remove access to the work immediately and investigate your claim 


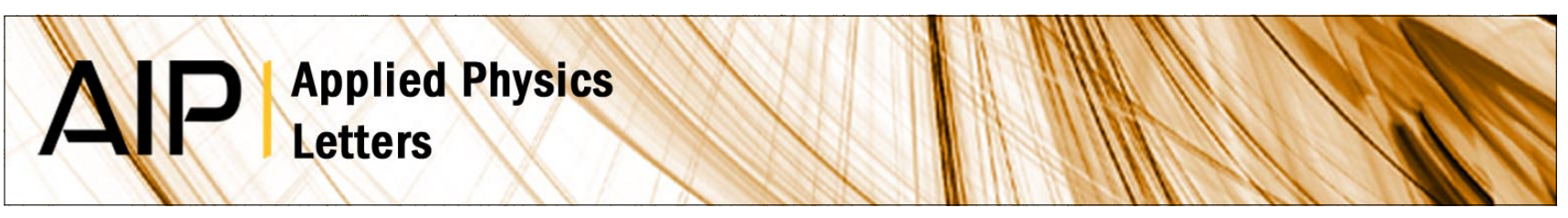

\section{Tunable true-time delay of a microwave photonic signal realized by cross gain modulation in a semiconductor waveguide}

Weiqi Xue and Jesper Mørk

Citation: Appl. Phys. Lett. 99, 231102 (2011); doi: 10.1063/1.3665946

View online: http://dx.doi.org/10.1063/1.3665946

View Table of Contents: http://apl.aip.org/resource/1/APPLAB/v99/i23

Published by the American Institute of Physics.

\section{Related Articles}

Low-loss flake-graphene saturable absorber mirror for laser mode-locking at sub-200-fs pulse duration Appl. Phys. Lett. 99, 261109 (2011)

Performance of large-area few-layer graphene saturable absorber in femtosecond bulk laser Appl. Phys. Lett. 99, 261107 (2011)

Mid-infrared quantitative spectroscopy by comb-referencing of a quantum-cascade-laser: Application to the $\mathrm{CO} 2$ spectrum at $4.3 \mathrm{~m}$

Appl. Phys. Lett. 99, 251107 (2011)

Theoretical study on phase locking of the array of fiber lasers coupled by bi-dimensional mutual injection AlP Advances 1, 042178 (2011)

Third harmonic generation by a low intensity laser pulse in a corrugated discharge capillary Appl. Phys. Lett. 99, 211501 (2011)

\section{Additional information on Appl. Phys. Lett.}

Journal Homepage: http://apl.aip.org/

Journal Information: http://apl.aip.org/about/about_the_journal

Top downloads: http://apl.aip.org/features/most_downloaded

Information for Authors: http://apl.aip.org/authors

\section{ADVERTISEMENT}






\title{
Tunable true-time delay of a microwave photonic signal realized by cross gain modulation in a semiconductor waveguide
}

\author{
Weiqi Xue ${ }^{\mathrm{a})}$ and Jesper Mørk \\ DTU Fotonik, Department of Photonics Engineering, Technical University of Denmark, Build. 343, \\ DK-2800 Kongens Lyngby, Denmark
}

(Received 17 September 2011; accepted 14 November 2011; published online 5 December 2011)

\begin{abstract}
We experimentally demonstrate the realization of a tunable true-time delay for microwave signals by exploiting cross gain modulation among counter-propagating optical beams in a semiconductor optical amplifier. Broadband operation from $\sim 5$ to $\sim 35 \mathrm{GHz}$ is observed. The physical effect originates from the combination of carrier dynamics and propagation effects, and the experimental results are well accounted for by a numerical model. We find that, in contrast to the case of the co-propagating beams, the bandwidth is not limited by the lifetime of excited carriers. The trade-off between the magnitude of the true-time delay and the microwave bandwidth is discussed. (C) 2011 American Institute of Physics. [doi:10.1063/1.3665946]
\end{abstract}

Since the demonstration of an optically steered phased array antenna in $1991,{ }^{1}$ the study of tunable microwave time delay lines realized by photonics has become a hot topic within the developing research area of microwave photonics. ${ }^{2}$ A common scheme exploits dispersion, with the time delay being varied by tuning the laser wavelength and propagating through high dispersion fibers ${ }^{3,4}$ or chirped fiber gratings. ${ }^{5-7}$ This scheme is, however, relatively bulky and complex. Recently, both slow light effects ${ }^{8}$ and microring resonators ${ }^{9}$ have been exploited to achieve microwave time delays. In particular, slow light in active semiconductor waveguides can provide very fast tuning speed, compact size, and low power consumption. ${ }^{10-13}$ Though microwave phase shifts beyond $360^{\circ}$ have been experimentally demonstrated, ${ }^{13}$ fundamental limitations ${ }^{11,14}$ make it difficult to achieve true time delays over a broad bandwidth, e.g., several tens of $\mathrm{GHz}$, by using slow light effects.

In this work, we demonstrate that microwave true-time delays can be realized in a semiconductor optical amplifier (SOA) by exploiting cross gain modulation (XGM) effects among two counter-propagating optical signals. It is wellknown that XGM in an SOA can be used to perform different optical signal processing functions. ${ }^{15-18}$ However, while the amplitude response of XGM has been extensively investigated, ${ }^{19,20}$ the phase response only received little attention. Experimentally, we find very different phase responses for co- and counter-propagating configurations. In particular, the counter-propagating scheme can induce true-time delays in a range up to $\sim 10$ ps over a microwave frequency band from a few $\mathrm{GHz}$ to $\sim 35 \mathrm{GHz}$, thus strongly exceeding the frequency limitation usually implied by the inverse of the carrier lifetime. The observations are accounted for by a numerical model, and a qualitative explanation is given.

Fig. 1(a) shows the experimental setup with the signal and probe beam co-propagating through an SOA. The wavelength of the $\mathrm{CW}$ probe beam is $1550 \mathrm{~nm}$, and it has a fixed optical power of $-5 \mathrm{dBm}$. The signal beam is modulated by a microwave signal generated by a network analyzer. The



modulation index is $\sim 4 \%$. The wavelength of the signal beam is $1540 \mathrm{~nm}$, and the optical power can be tuned by a variable optical attenuator. Both beams are TE polarized. The SOA is electrically biased at $340 \mathrm{~mA}$, at which current the measured carrier lifetime is $\sim 60 \mathrm{ps}$. After the SOA, the probe beam is selected by an optical bandpass filter and detected by the network analyzer. For the counterpropagating configuration, the probe beam is selected by an optical circulator, as shown in Fig. 1(b). Four-wave mixing effects between the signal and probe beams can be neglected due to the large detuning.

The measured XGM response as a function of the microwave modulation frequency for different signal laser power levels is shown in Fig. 2. In order to clearly compare the slopes, the response curves are shifted to 0 by their value at the lowest modulation frequency. For the co-propagating scheme shown in Fig. 2(a), a resonance-like peak appears at a few $\mathrm{GHz}$, which has been shown to originate from the nonzero value of the internal loss. ${ }^{20-22}$ As the microwave frequency increases, the XGM responses appear almost parallel, implying only a small change of group velocity and time delay with power. In contrast, for the counter-propagating scheme, the slope of the XGM response shows large changes

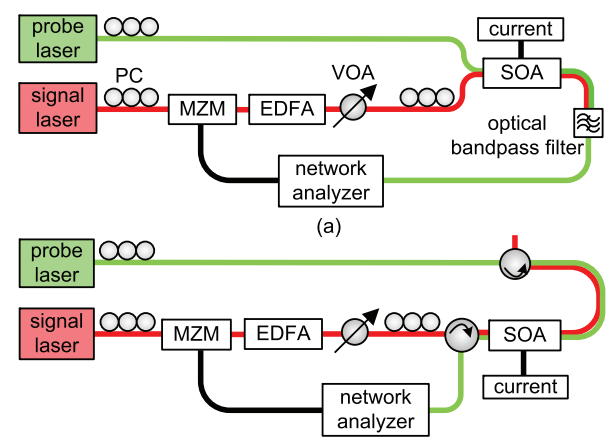

(b)

FIG. 1. (Color online) Experimental set-ups for measuring the microwave time delay and power response induced by an SOA for (a) co- and (b) counter-propagating configurations. PC: polarization controller. MZM: Mach-Zehnder intensity modulator. EDFA: Erbium doped fiber amplifier. VOA: Variable optical attenuator. 


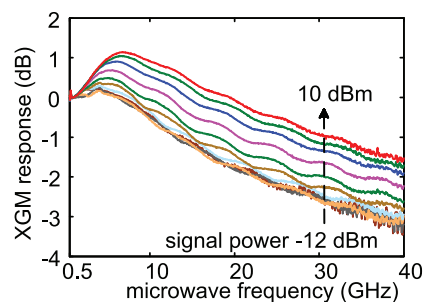

(a)



(b)
FIG. 2. (Color online) Measured XGM amplitude response versus microwave frequency for different optical signal power levels in the (a) co- and (b) counter-propagating configurations.

with signal power, cf., Fig. 2(b). The fast drop-off of the XGM response seen in this case is caused by the transit time effect, i.e., phase mismatch, between the counterpropagating signal and the probe beams. ${ }^{21}$ Therefore, it is expected that these optically induced slope changes in the frequency range from $\sim 5$ to $40 \mathrm{GHz}$ imply large time delays for the microwave signal carried by the probe beam.

The measured microwave time delays shown in Fig. 3 are relative to a reference case, where the input signal power is the minimum, i.e., $-12 \mathrm{dBm}$ for the co-propagating configuration and $-5 \mathrm{dBm}$ for the counter-propagating configuration. The two configurations are seen to result in qualitatively different responses. For the co-propagating case (Fig. 3(a)) the induced time delay rapidly decreases with frequency, showing the usual lifetime limitation. These results share common features with slow light in SOAs mediated by coherent population oscillations. ${ }^{10-12}$ In contrast, for the counter-propagating configuration (Fig. 3(b)) a tunable truetime delay from 0 to $\sim 10 \mathrm{ps}$ is obtained over a large range of frequencies, extending from $\sim 5$ to $35 \mathrm{GHz}$ by tuning the input optical signal power.

We now briefly describe the theoretical model used to analyze the experimental results. Restricting attention to small-signal and harmonic modulation, the power of the signal and probe beams, $P_{s}$ and $P_{p}$, can be expressed ${ }^{22}$

$$
\left\{\begin{array}{l}
P_{s}(z)=\bar{P}_{s}(z)+\Delta P_{s}(z) \cdot e^{-i \Omega t}+\Delta P_{s}^{*}(z) \cdot e^{i \Omega t} \\
P_{p}(z)=\bar{P}_{p}(z)+\Delta P_{p}(z) \cdot e^{-i \Omega t}+\Delta P_{p}^{*}(z) \cdot e^{i \Omega t}
\end{array}\right.
$$

where $\bar{P}_{m}(z),(m=s$ or $p)$ is the average optical power, which is a function of the propagation distance $z$ inside the SOA. $\Delta P_{m}(z)=\left|\Delta P_{m}\right| \times \exp \left(i k_{m} z\right)$ is the modulated component at microwave frequency $\Omega, k_{m}=\Omega / v_{m}$ is the propagation constant of the modulation component, and $v_{m}$ is the phase velocity. If we assume the probe beam to be a weak perturbation, the gain dynamics and the propagation of the signal beam in the SOA will not be impacted by the probe beam. ${ }^{22}$ Then, the propagation equations for $\Delta P_{m}(z)$ in both co- and counter-propagating configurations can be described by

$$
\begin{gathered}
\frac{d \Delta P_{s}}{d z}=(g-a) \cdot \Delta P_{s}-\frac{g \bar{P}_{s} / P_{s a t}}{1+\bar{P}_{s} / P_{s a t}-i \Omega \tau} \cdot \Delta P_{s} \\
\frac{d \Delta P_{p}}{d z}=(g-a) \cdot \Delta P_{p}-\frac{g \bar{P}_{p} / P_{s a t}}{1+\bar{P}_{s} / P_{s a t}-i \Omega \tau} \\
\times\left[\Delta P_{s} \cdot \exp (i \Delta k z)+\Delta P_{p}\right],
\end{gathered}
$$

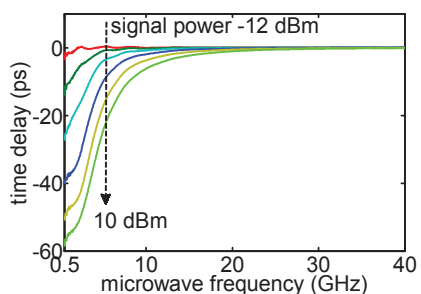

(a)

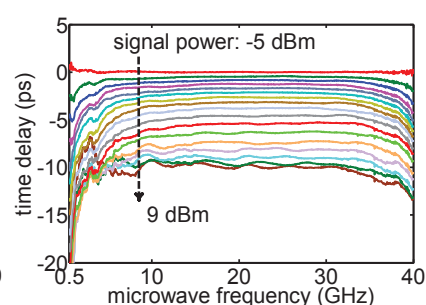

(b)
FIG. 3. (Color online) Measured microwave time delay versus microwave frequency for different optical signal power levels in the (a) co- and (b) counter-propagating configurations.

where $P_{\text {sat }}$ is the saturation power of the SOA, $a$ is the internal loss, $\tau$ is the carrier lifetime, and $g$ is the saturated gain. Propagation effects are taken into account by the term $\exp (i \Delta k z){ }^{22}$ For the counter-propagating configuration

$$
\Delta k=k_{s}-k_{p}=\frac{\Omega}{v_{s}}-\frac{\Omega}{v_{p}}=-\left(n_{s}+n_{p}\right) \frac{\Omega}{c}=-2 n \frac{\Omega}{c} .
$$

Here, $c$ is the speed of light speed in vacuum. For the co-propagating configuration, $\Delta k=0$.

The boundary conditions are $\Delta P_{s}(0)=\Delta P_{\text {in }}$ and $\Delta P_{p}(0)=0$ for co-propagating configuration and $\Delta P_{s}(0)=\Delta P_{i n}$ and $\Delta P_{p}(L)=0$ for counter-propagation. Here, $\Delta P_{\text {in }}$ is the input modulated component of the signal beam and $L$ is the length of the SOA. Based on Eq. (2) and these boundary conditions, the microwave modulation imposed by the signal on the probe can be calculated, therefore, the induced microwave time delays can be inferred. The parameters used for the calculations are $\tau=100 \mathrm{ps}$, $L=1 \mathrm{~mm}, P_{\text {sat }}=5 \mathrm{~mW}, g=1.5 \times 10^{2} \mathrm{~cm}^{-1}, a=13.5 \mathrm{~cm}^{-1}$, and refractive indices $n_{s}=n_{p}=n=3.5$. Figure 4 shows the calculated XGM responses for the co- and counterpropagating configurations. The simulated results are seen to agree very well with the experimental measurements depicted in Fig. 2. One can understand these effects as a combination of carrier dynamics and propagation effects. In the counter-propagating case, due to the phase mismatch between the carrier oscillations experienced by the probe beam and the modulated component of the signal beam, as displayed by the term $i \Delta k z$ in Eq. (2b), the XGM response decreases much faster with microwave frequency as compared to the co-propagating case. The dip of the XGM response occurring in Fig. 4(b) at a frequency of $\sim 43 \mathrm{GHz}$ corresponds to the frequency, at which the length of the SOA equals half a microwave wavelength

$$
\frac{\Omega}{2 \pi}=\frac{c}{2 n L}=\frac{3 \times 10^{8} \mathrm{~m} / \mathrm{s}}{2 \times 3.5 \times 1 \mathrm{~mm}} \approx 43 \mathrm{GHz} .
$$

This value is just outside the frequency range that is experimentally accessible with our network analyzer.

Figure 5 shows the calculated microwave time delays. In the measured frequency range, the theory shows good agreement with the measurements in Fig. 3. Let us discuss briefly the phase response for the counter-propagating configuration. When the microwave frequency approaches the dip frequency of $43 \mathrm{GHz}$, the phase approaches $180^{\circ}$ for the largest power level, which is the generic phase response if one considers the 


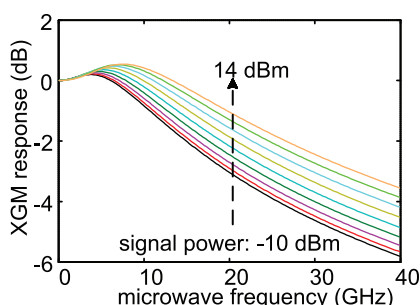

(a)

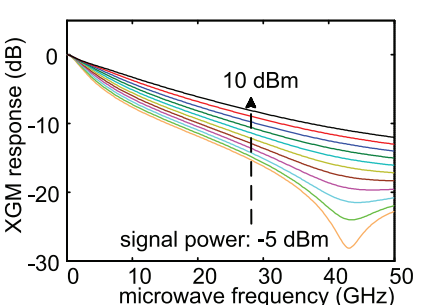

(b)
FIG. 4. (Color online) Calculated XGM responses versus microwave frequency for different optical signal power levels in the (a) co- and (b) counter-propagating configurations.

XGM response as a microwave notch filter profile. ${ }^{9}$ At low microwave frequencies, because the length of the SOA is much smaller than the wavelength of the microwave modulation, propagation effects inside the SOA can be neglected. Hence, in the low-frequency range, the obtained time delay will be the same for the co- and counter-propagating configurations and is dominated by dynamical gain saturation effects. ${ }^{14}$ In the intermediate microwave frequency range, i.e., from a few $\mathrm{GHz}$ to $\sim 35 \mathrm{GHz}$, the counter-propagating XGM amplitude and phase responses are nearly linear, and their slopes can be changed by varying the input signal power, as shown in Figs. 2(b) and 4(b), thus corresponding to a constant time delay.

The maximum obtained time delay $\Delta t_{R F}$ is limited by the transit time through the SOA

$$
\Delta t_{R F}=\frac{n L}{c} .
$$

The microwave bandwidth $\Delta f_{R F}$ over which the delay appears as a true-time delay is approximately given by the difference between the cavity frequency determined by Eq. (3) and the inverse of the carrier lifetime

$$
\Delta f_{R F} \approx \frac{c}{2 n L}-\frac{1}{\tau} .
$$

As suggested by Eq. (4a), for the counter-propagating based XGM scheme, the maximum achievable true-time delay can be extended by increasing the length of the SOA. However, from Eq. (4b), it is apparent that there is a trade-off between the microwave operation bandwidth and the induced time delay. For applications requiring a signal bandwidth of several hundreds of $\mathrm{MHz},{ }^{23}$ even a few $\mathrm{GHz}$, a tunable true time delay of several tens of picoseconds at $\sim 10 \mathrm{GHz}$ can still be achieved by properly designing the length and carrier lifetime of the SOA.

In conclusion, we report distinctly different phase responses for co- and counter-propagating XGM schemes in active semiconductor waveguides. For the co-propagating configuration, gain dynamics leads to time delays of several tens of picoseconds but restricted to a rather low frequency range. In contrast, for the counter-propagation case, due to the linear variation of the phase with microwave frequency, a $\sim 10$ ps tunable true-time delay over a microwave band-

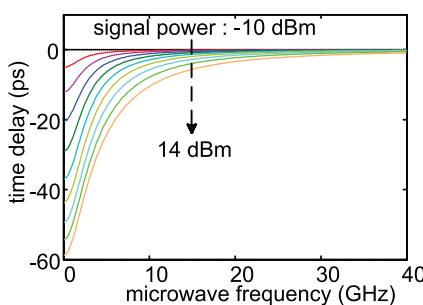

(a)

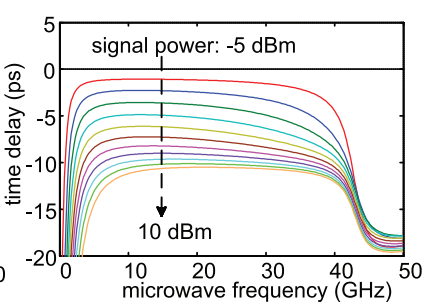

(b)
FIG. 5. (Color online) Calculated microwave time delays versus microwave frequency for different optical signal power levels in the (a) co- and (b) counter-propagation configurations.

width of several tens of $\mathrm{GHz}$ is achieved. For both configurations, theoretical simulations based on a rate equation model for the carrier density and propagation equations for the intensity of the beams account very well for the experimental results. The models can be used to further optimize the obtained time delay. By properly engineering the length and carrier lifetime of the active semiconductor waveguide, a tunable true-time delay of several tens of picoseconds should be achievable.

The authors would like to acknowledge support from the European Commission via the FP7 project "GOSPEL" as well as the Danish Research Councils via the project "QUEST."

${ }^{1}$ W. Ng, A. A. Walston, G. L. Tangonan, J. Lee, I. L. Newberg, and N. Bernstein, J. Lightwave Technol. 9, 1124 (1991).

${ }^{2}$ J. Capmany and D. Novak, Nature Photon. 1, 319 (2007).

${ }^{3}$ O. Raz, R. Rotman, Y. Danziger, and M. Tur, IEEE Photon. Technol. Lett. 16, 1367 (2004).

${ }^{4}$ B. Juswardy, F. Xiao, and K. Alameh, Opt. Express 17, 4773 (2009).

${ }^{5}$ J. L. Corral, J. Marti, J. M. Fuster, and R. I. Laming, IEEE Photon. Technol. Lett. 9, 1529 (1997).

${ }^{6}$ B. Ortega, J. L. Cruz, J. Capmany, M. V. Andres, and D. Pastor, J. Lightwave. Technol. 18, 430 (2000).

${ }^{7}$ Y. Liu, J. Yang, and J. Yao, IEEE Photon. Technol. Lett. 14, 1172 (2002).

${ }^{8}$ M. Bashkansky, Z. Dutton, A. Gulian, D. Walker, F. Fatemi, and M. Steiner, Proc. SPIE 7226, 72260A (2009).

${ }^{9}$ M. Pu, L. Liu, W. Xue, Y. Ding, H. Ou, K. Yvind, and J. M. Hvam, Opt. Express 18, 6172 (2010).

${ }^{10}$ C. J. Chang-Hasnain and S. L. Chuang, J. Lightwave Technol. 24, 4642 (2006).

${ }^{11}$ A. V. Uskov, F. G. Sedgwick, and C. J. Chang-Hasnain, IEEE Photon. Technol. Lett. 18, 731 (2006).

${ }^{12}$ J. Mørk, F. Öhman, M. van der Poel, Y. Chen, P. Lunnemann, and K. Yvind, Laser Photon. Rev. 3, 30 (2009).

${ }^{13}$ W. Xue, S. Sales, J. Capmany, and J. Mørk, Opt. Express 18, 6156 (2010).

${ }^{14}$ J. Mørk, R. Kjær, M. van der Poel, and K. Yvind, Opt. Express 13, 8136 (2005).

${ }^{15}$ T. Durhuus, B. Mikkelsen, C. Joergensen, S. L. Danielsen, and K. Stubkjær, J. Lightwave Technol. 14, 942 (1996).

${ }^{16}$ K. E. Stubkjær, IEEE J. Sel. Top. Quantum Electron. 6, 1428 (2000).

${ }^{17}$ H. J. S. Dorren, M. T. Hill, Y. Liu, N. Calabretta, A. Srivatsa, F. M. Huijskens, H. de Waardt, and G. D. Khoe, J. Lightwave Technol. 21, 2 (2003).

${ }^{18}$ Q. Wang, F. Zeng, S. Blais, and J. Yao, Opt. Lett. 31, 3083 (2006).

${ }^{19}$ D. A. O. Davies, IEEE Photon. Technol. Lett. 7, 617 (1995).

${ }^{20}$ A. Mecozzi, IEEE Photon. Technol. Lett. 8, 1471 (1996).

${ }^{21}$ M. L. Nielsen, D. J. Blumenthal, and J. Mørk, J. Lightwave Technol. 18, $2151(2000)$

${ }^{22}$ J. Mørk, A. Mecozzi, and G. Eisenstein, IEEE J. Sel. Top. Quantum Electron. 5, 851 (1999).

${ }^{23}$ H. Hashemi, X. Guan, A. Komijani, and A. Hajimiri, IEEE Trans. Microwave Theory Tech. 53, 614 (2005). 